\title{
Coupling Early Warning Services, Crowdsourcing, and Modelling for Improved Decision Support and Wildfire Emergency Management
}

\author{
C.Bielski*, V.O’Brien*, C.Whitmore*, K.Ylinen ${ }^{\dagger}$, I.Juga ${ }^{\dagger}$, P.Nurmi ${ }^{\dagger}$, J.Kilpinen ${ }^{\dagger}$, I.Porras ${ }^{\ddagger}$, J.M.Sole ${ }^{\ddagger}$, P.Gamez $^{\ddagger}$, M.Navarro ${ }^{\ddagger}$,

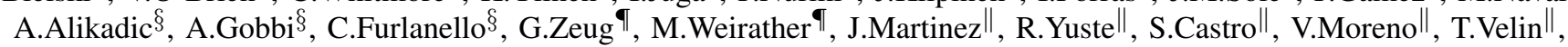 \\ and C.Rossi** \\ *EOXPLORE UG, Weil am Rhein, Germany \\ Email: conrad.bielski@eoxplore.com \\ $\dagger^{\dagger}$ Finnish Meteorological Institute (FMI), Helsinki, Finland \\ $\ddagger_{\text {METEOSIM S.L., Barcelona, Spain }}$ \\ $\S$ Predictive Models for Biomedicine \& Environment, Fondazione Bruno Kessler (FBK), Povo (Trento), Italy \\ "Terranea UG, Geltendorf, Germany \\ ${ }_{\text {Answare Tech S.L, Murcia, Spain }}$ \\ ** Istituto Superiore Mario Boella (ISMB), Torino, Italy
}

\begin{abstract}
The threat of a forest fire disaster increases around the globe as the human footprint continues to encroach on natural areas and climate change effects increase the potential of extreme weather. It is essential that the tools to educate, prepare, monitor, react, and fight natural fire disasters are available to emergency managers and responders and reduce the overall disaster effects. In the context of the I-REACT project, such a big crisis data system is being developed and is based on the integration of information from different sources, automated data processing chains and decision support systems. This paper presents the wildfire monitoring for emergency management system for those involved and affected by wildfire disasters developed for European forest fire disasters.
\end{abstract}

Keywords-wildfire; monitoring; modeling; emergency management; analytics;

\section{INTRODUCTION}

The consensus is that the frequency and severity of extreme weather events around the globe due to climate changes will increase [1] effectively increasing the chances of natural disasters such as flooding and wildfires ${ }^{1}$. In the case of wildfire disasters, they are produced by weather events such as heatwaves and droughts. In Europe, wildfires are being recorded in regions where they were uncommon or non-existent. Furthermore, wildfires also contribute significantly to greenhouse gases that drive global warming.

Model predictions show that wildfire seasons will be longer, produce more smoke, and burn larger areas by 2050 [2], [3]. In Europe, the fire Seasonal Severity Rating (SSR) projected based on Regional Climate Model data show similar trends where the area at risk of fire danger will increase [4].

\footnotetext{
${ }^{1}$ https://www.ipcc.ch/pdf/assessment-report/ar5/wg2/WGIIAR5Chap23_FINAL.pdf
}

The risk of wildfire disaster will spread across Europe into areas where it was not considered a threat before. Local and regional authorities as well as the general public will need to take this new danger into consideration and be prepared to deal with it. The goal of the I-REACT project (http://www.i-react.eu/) is to provide the technical tools in order to 1) educate the public about natural disasters and take advantage of crowdsourced information, 2) provide automated monitoring, modeling and alerting services for specific types of natural disasters and 3) when disaster strikes, share the information with the relevant emergency responders and managers to help make decisions and plan an appropriate response that can minimise the threats to human life and economic damages. I-REACT is funded by an H2020 EU grant and this article presents the system being developed specifically to combat European wildfire disasters. Within the wildfire monitoring system there is a demand for access to relevant information that can be used in the context of wildfire disasters including early warnings, forecasts, extreme weather conditions, seasonal climate forecasts, and risk assessments through geospatial risk maps, social media analysis, and decision support system models. This paper presents the big data architecture built to support the data and information analysis system to provide novel wildfire monitoring capabilities to emergency managers.

\section{Methodology}

The methodology section is made up of two parts. The first part presents the overall system design demonstrating the varied data sets being ingested and processed. The second part describes the information produced by the wildfire monitoring system to support emergency management. 


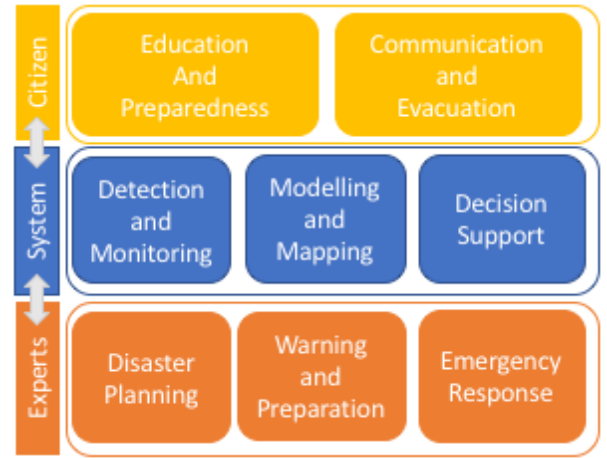

Figure 1. The three components driving the emergency management system and what they represent.

\section{A. Data Processing and Analysis Chains}

Feedback mechanisms are at the heart of the system because when new data and information is made available, they should be integrated into the analysis to improve situational awareness and decision making. The three key components are the citizen, the system, and the expert. These three components are paramount (Figure 1) because of the fact that this emergency management system was designed for natural disasters in general and not only wildfires.

To better understand the architecture implemented for the wildfire system, figure 2 presents a Business Process Modeling Notation (BPMN) diagram. Due to the fact that the authorities and/or experts involved in the emergency services are the ones that make all the final decisions, it is important to share all updated information from citizens, social media, EO, and the different models with them. Finding a balance based on their emergency response operational plans and the different types of information gathered and produced by the system can be tuned through the decision support system. However, a wildfire emergency activation/de-activation is fully under the control of the authorities as well as any communications to the affected population during a disaster.

The big data analysis and machine learning architecture that was designed for the system is too complex to include in this article. However, it should be noted that without the modular system that was implemented, the different disaster emergency types, i.e. not just for wildfire disasters and situations, could not be easily adapted to all work within the same framework.

\section{B. Processing Description}

1) Weather Forecasting: The risk of wildfire disasters increases when regional conditions become favourable for fire. Monitoring long term weather is one of the ways to provide insight into the regional risk of wildfire. In order to do this, the Finnish Meteorological Institute (FMI) provides European scale ensemble forecasts that are used to calculate the Fire Weather Index. Ensemble prediction systems consist of multiple separate numerical weather predictions called members. All members have slightly different initial conditions, different forecast models, or different formulations of forecast models. Ensemble forecasting is used to account for the inherent uncertainty of weather forecasts.

The ensemble forecasts are produced by the European Centre for Medium-range Weather Forecasts (ECMWF) and provide longer-term forecasts from a few days to a couple of weeks. The ECMWF ensemble consists of 51 members and a spatial resolution of 18 kilometers. These forecasts are updated twice daily at $00 \mathrm{~h}$ and $12 \mathrm{~h}$. An finer $8 \mathrm{~km}$ spatial resolution optional model called the Grand Limited Area Model Ensemble Prediction System (GLAMEPS) having 8 $\mathrm{km}$ can be used for short term forecasts ranging from a few hours to a couple of days. The GLAMEPS has 52 members and forecasts are updated four times a day. The forecast variables of interest to the wildfire system are temperature, dew point temperature, wind speed, precipitation, as well as accumulated precipitation analysis for the previous day.

Due to the fact that initial ensemble forecasts tend to be under dispersive and biased, they are calibrated (both ECMWF and GLAMEPS) to produce more reliable and useful forecasts especially for adverse weather events. Statistical and numerical methods are used with the information from prior forecasts and observations to calibrate and improve the predictions. The calibration is useful at a variety of forecast time scales including the lead times from few hours [5] to two weeks [6].

2) The Fire Weather Index: In order to provide information on the regional conditions relevant to the risk of wildfire, the Fire Weather Index (FWI) is calculated based on the continuously updated weather forecast. The FWI calculated by Meteosim S.L. across Europe is the Canadian Forest Fire Weather Index ${ }^{2}$ that is applied worldwide.

Using the ECMWF and GLAMEPS ensemble forecasts, this well known fire danger rating index is computed. The calculation is produced based on the following input data and indices: Fine Fuel Moisture Code (FFMC), Duff Moisture Code (DMC), Drought Code (DC), Initial Spread Index (ISI), Buildup Index (BUI), and the Daily Severity Rating (DSR). This is computed for each of the ensemble members, i.e. 51 ECMWF based FWI ensemble forecasts and 52 GLAMEPS based FWI ensemble forecasts outputting FWI at the same spatial resolution and lead times as the input ensemble forecasts. Note that slightly different initial temperature, dew point temperature, wind speed, and 24hour precipitation values output different FWI values across Europe.

3) Monitoring Hotspots: When the risk of wildfire is high based on the above mentioned FWI, fires can be started both naturally and anthropogenically. However, the only way to monitor such vast areas at continental and global scales

\footnotetext{
${ }^{2}$ http://cwfis.cfs.nrcan.gc.ca/background/summary/fwi
} 


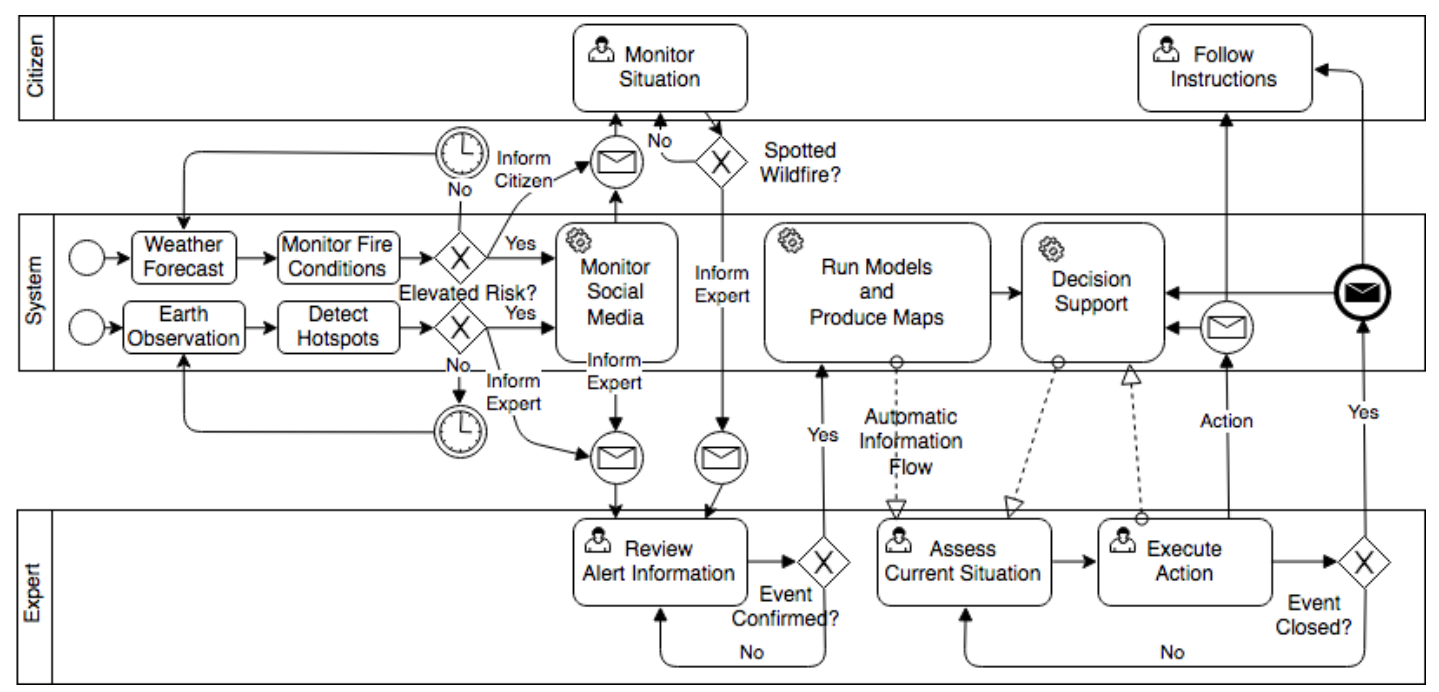

Figure 2. Flow of the proposed wildfire monitoring and emergency management service in BPMN. The goal of the system is to continuously provide updated information to both the authorities (expert) and the citizen.

for active fires is with satellite based Earth Observation (EO). For the current iteration of the wildfire monitoring service, two EO products are used to identify hot spots across Europe: one based on the Visible Infrared Imaging Radiometer Suite (VIIRS) [7] and the other on the Moderate Resolution Imaging Spectroradiometer (MODIS) [8].

Spotting hot spots across Europe with EO produces quite coarse spatial resolution data and furthermore, just because a pixel appears 'hot' does not mean that it is necessarily due to a fire. Therefore, the input data is processed based on the number of hotspots detected and grouped by NUTS $^{3}$ level 3 (region) and 5 (commune). This provides a more regional overview where potential fires are raging so that they can be shared with the citizen, system, and experts.

To be certain that the EO derived hot spot data is accurate, the areas identified are provided to the citizens located in that area to look out for and maybe confirm wildfires, the social media monitoring engine can listen for mention of wildfire within specific regions, and the authorities can be warned that potential fires have been identified in their area of operations.

4) Confirmation of a Wildfire: If and when there is information arriving into the system that a wildfire has been identified, it is up to the local authorities to trigger an emergency response. When such a confirmation has been received by the system, then new processes are automatically started related to the continued monitoring of the wildfire affected area, running wildfire nowcast and forecast models, producing risk maps based on up-to-date information, and keeping an eye on social media and affected citizens.

\footnotetext{
${ }^{3}$ NUTS - Nomenclature of Territorial Units For Statistics http://ec.europa.eu/eurostat/web/nuts .
}

5) Wildfire Hazard Nowcasting and Forecasting: In the case of a wildfire disaster, the first responders need not only to plan how best to evacuate the affected population but firefighters also need to plan how to attack and extinguish the fire as quickly as possible. For this reason, wildfire nowcasting and forecasting maps are produced based on the latest weather forecasts provided by the service partners. A Rate of Spread (ROS) model based on the U.S. Forest Service BEHAVE system [9], [10] is used in the context of this service. Wildfire spread models require a number of inputs to produce a viable fire spread output. The following input datasets are used:

- European Fuel Map - JRC product containing the National Forest Fire Laboratory standard fuel models defined by the USDA Forest Service ${ }^{4}$;

- European Digital Elevation Model (EU-DEM) - a panEuropean open access elevation model used for elevation, slope and aspect information ${ }^{5}$;

- Fuel Moisture - extracted from the FWI information;

- Wind direction and Speed - extracted from the weather forecast;

- Location of the point of ignition - either from EO hotspot data and/or the authorities.

With all of the input data in-hand, it is possible to produce wildfire nowcast maps that span from the date and time of the forecast up to 48 hours. Forecasting of the wildfire is between 48 hours and 72 hours. Greater lead times for the forecast are not being produced because of the changing burned area extent and weather which can significantly

\footnotetext{
${ }^{4}$ JRC Contract Number 384347 on the "Development of a European Fuel Map".

${ }^{5}$ Copernicus DEM over Europe https://www.eea.europa.eu/data-andmaps/data/eu-dem .
} 
Table I

THE DATA USED For THE INFRASTRUCTURES DATA. SOURCE: OpEN STREET MAP (OSM) (PROVIDED BY TERRANEA), NATIONAL DATA, AND UNESCO.

\begin{tabular}{|c|c|c|}
\hline SECTOR & TYPE & SOURCE \\
\hline \hline \multirow{2}{*}{ Energy } & Powerplants & OSM + national data \\
& Pipelines & OSM \\
& Oowerlines & OSM \\
\hline \multirow{2}{*}{ Transportation } & Bridges & OSM \\
& Airports & OSM \\
\hline Schools & Railway & OSM \\
\hline Water & Schools & OSM \\
\hline Health Care & Wastewater & OSM \\
& Water supply & OSM + national data \\
\hline \multirow{2}{*}{ Administration } & Pharmacies & OSM + national data \\
& Hospitals & OSM + national data \\
\hline Telecom Towers & Police Station & OSM \\
\hline Heritage Sites & UNESCO Heritage Sites & OSM \\
\hline
\end{tabular}

alter wildfire spread. The maps are produced automatically whenever an updated weather forecast has been ingested into the system.

6) Wildfire Risk Impact Mapping: During an active wildfire disaster, the emergency responders require knowledge about the potential risk affecting the local population. The Fondazione Bruno Kessler (FBK) produce risk impact raster maps computed using a fuzzy methodology pipeline [11]. The nowcast and forecast wildfire hazard maps (described above) are used as the exposure layer for the risk impact assessment. The sensitivity layers, which are the impact affected by the wildfire are the following: total population, population under 5 years of age, population over 65 years of age, and critical infrastructures.

The population grid for the year 2016 was derived from the $\mathrm{JRC}^{6}$ data product having a spatial resolution of $100 \mathrm{~m}$. Using the EUROSTAT regional statistics for NUTS $3^{7}$, the age group layers (under 5 and over 65 of age) were computed based on the hypothesis that the percentage of younger and elder is constant in the corresponding NUTS region.

The infrastructure data was acquired and prepared from Open Data sources by Terranea (see table I). For each infrastructure type in table I, a raster was generated such that each pixel value was computed as the ratio between the number of infrastructures in the cell and the total number of that type of infrastructure. The spatial resolution of the raster layer was aligned with the population raster. The infrastructure layer used in the fuzzy model was the

\footnotetext{
${ }^{6} \mathrm{JRC} \quad \mathrm{http}: / /$ data.jrc.ec.europa.eu/dataset/jrc-ghslghs_pop_eurostat_europe_r2016a

${ }^{7}$ http://ec.europa.eu/eurostat/web/regions/data/database
}

mean (or sum since the fuzzy model is invariant by scalar multiplication) of all the layers. Note that this approach can be adapted in case the user wants to include more details in some of the model layers with respect to others, e.g. using a weighted mean. The results of the wildfire risk impact maps are shared with the authorities together with the wildfire nowcast and forecast maps. The risk impact maps are also automatically updated when new wildfire nowcast and forecast maps are produced.

7) Wildfire Disaster Decision Support: The Decision Support System (DSS) module is a tool that integrates user action plans with respect to different natural disaster emergencies. This tool offers suggestions about field missions, alerts, warnings, and requesting reports from the different actors involved in responding to a crisis. The DSS helps the authorities to make decisions based on an agreed protocol, information generated by the other service modules as well as Open Data already available in the system, e.g. critical infrastructures to improve response times and the transmission of warnings, alerts and other information. The implemented DSS is a Java and Python application that uses REST API services and publishes/subscribes protocols to communicate within the system.

The DSS is integrated into the system and receives information from external modules through internal system modules (unfortunately more details of the system architecture and back-end design are beyond the scope of this article). Any new crisis knowledge can be sent to the service back-end where it can then be visualised through the user interface. The DSS data flow is based on receiving messages from the system having wildfire situational information and reacting to them. When the DSS receives a new message through the systems messaging services, the DSS reads the message and retrieves the information to which the message is referring. Having received a set of wildfire related situational information (as described above), the DSS compares the information with the authorities crisis plan that has been implemented as a set of rules. This was achieved using a Rules Engine - Drools (https://www.drools.org/). Based on the crisis action plans and the wildfire information provided by the system, the DSS generates a set of suggestions related to: alerts, warnings, missions, and/or report requests to help support authorities making decisions during the crisis. The supporting suggestions must be either approved, rejected or modified by the decision maker because the system will not automatically carry out any of the suggestions. All DSS suggestions are presented via the system front-end but are also available to the back-end for other modules.

8) Burned Area Mapping: Wildfire disaster events can affect large areas that need to be mapped in order to assess and evaluate economic and environmental damages, to model trace gas emissions, and to plan for the landscape recovery. Burned area maps are also important to feed-back into the wildfire nowcast and forecast maps. Furthermore, to 
prepare and mitigate for the future it is necessary to learn from past events in order to understand drivers and risks and to identify actionable recommendations to enhance disaster resilience [12]. Remote sensing is the standard tool used to delineate the affected burnt area. As part of the MODIS Active Fire and Burned Area Products, burned areas are mapped at global scale investigating spectral, temporal, and structural changes [13]. The application of various spectral indices such as the Normalised Difference Vegetation Index (NDVI), Burned Area Index (BAI), and Normalized Burn Ratio (NBR) have been widely used to monitor fire-induced vegetation changes [14], [15].

Within the wildfire system, the burned area mapping will be automated and based on finer spatial resolution EO imagery. When a wildfire disaster activation is received, different EO image databases will be searched for viable imagery based on the location information. The Copernicus Open Access Hub of the European Space Agency (ESA) ${ }^{8}$ can provide very fine spatial resolution Copernicus Sentinel2 optical imagery that is perfect for burned area mapping. The burned area maps produced will be sent to the system to be used for modeling and/or post-disaster analysis.

9) Citizen Involvement: It is imperative that citizens are kept away from wildfire disasters. However, they can also provide vital situational information when in proximity of such events through their mobile personal devices, which have become a commodity and always connected thanks to the ubiquity of cellular networks.

Similarly to [16], we propose to implement a specific mobile application to:

1) Increase citizens? awareness related to wildfires: users can be invited to build up their knowledge on wildfires in a "passive" manner, e.g. by reading tips, as well in an "active" mode, e.g. by answering quizzes;

2) Get informed about wildfire risk, warning and alerts: periodically produced fire hazard (e.g. FWI) and risk layers can be easily visualized in the mobile application through map layers, which should be delivered through OGC services (e.g. WMTS) in order to minimize bandwidth consumption. At the same time, asynchronous warnings and alerts produced by the authorities can be send to mobile devices in proximity of the area of interest of such communications through push notifications;

3) Produce real-time infield reporting: allowing citizens to send real-time geolocalized reports containing a picture along with vital information for wildfire detection and management e.g., fire or smoke presence and location, smoke color, flame heights, wind direction and wind speed. Of course some of such information should be qualitative because mobile devices does not include environmental sensors (e.g for wind direction

\footnotetext{
${ }^{8}$ https://scihub.copernicus.eu/
}
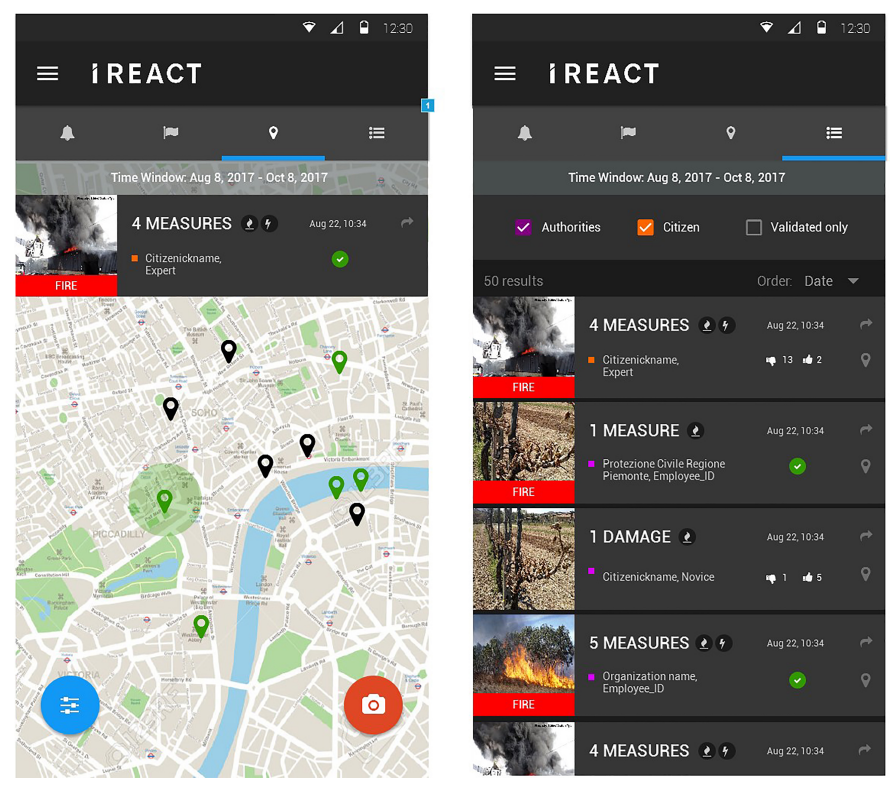

Figure 3. A preliminary view of the mobile app developed for use by citizens. The views provide some insight into the possibilities of citizens providing information on wildfire situation in their area (if safe to do so).

and speed).

4) Validate information: the mobile application can show to users early warning information (e.g., hot spot detection) and pre-labelled social media posts, asking them to validate such information.

The mobile application should be designed in order to provide a view of what is happening around the user (mobile device), i.e. within a given radius from the current location as provided by the GPS (we assume an outdoor context away from large urban centres). In this manner, users? will be able to provide reports of phenomena happening, and validate information generated in their proximity. The mobile application must distinguish between the position of the device, and the position of the reported event. This can be achieved by allowing the user to pinpoint on a map the location of the reported information.

In Figure 3, we present preliminary mock-ups of such a mobile application, with a focus on the reporting capability.

The goal of the methodology section was to demonstrate the variety and diversity of data and information as well as the partnerships needed to develop such a complex system as the presented wildfire monitoring system. Furthermore, the complexity of the possible analysis will only continue to increase as access to better data and information as well as improvements in models and processes are developed.

\section{RESULTS}

The following section presents a sample of the types of results being produced by the wildfire monitoring system for emergency management. Note that the system is still under active development using an Agile approach and therefore 


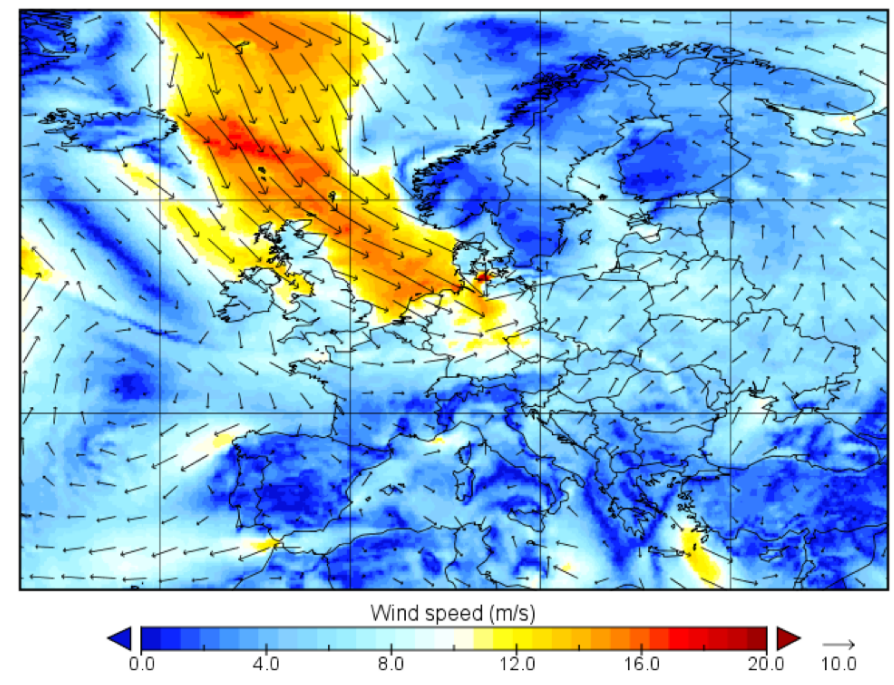

Figure 4. Wind speed $(\mathrm{m} / \mathrm{s})$ and direction forecast (at $10 \mathrm{~m}$ above ground) based on ECMWF ensemble model (one member).

continued discussions with users and managers will influence the model outputs as well as decision support system procedures. Due to publication constraints, only some of the results are presented and described here.

Weather forecasts are vital to wildfire monitoring and modeling. One variable that is provided for wildfire weather calculation is the wind forecast. It consists of $\mathrm{u}$ and $\mathrm{v}$ components (eastward and northward wind) from which the wind speed and direction can be calculated (figure 4). This information is not only used for the FWI computation but also needed for modeling wildfire spread.

Potential identification of wildfires can be identified based on EO imagery. Figure 5 shows the location of the identified hot spots based on VIIRS data and the communes in which they are located. Each polygon provides the place names that can be used for monitoring of social media channels. The colours reflect the number of identified hot spots within the region. This information is updated daily and automatically and provides the triggers to warn the authorities of potential dangers. Together with the FWI that provides information about the wildfire conditions in the region, they are a good indicator for wildfire hazards. Note that in the near future it is expected that a number of newer EO satellites will help in the identification of wildfire hot spots. This includes the currently orbiting European Copernicus Sentinel-3 satellite as well as the upcoming American (Q4 2017) Joint Polar Satellite System (JPSS), a collaboration between the National Oceanic and Atmospheric Administration (NOAA) and the National Aeronautics and Space Administration (NASA). It is expected that their input will be ingested into the wildfire monitoring system as well.

Once a wildfire disaster has been identified, the wildfire nowcast and forecast service begins producing maps based
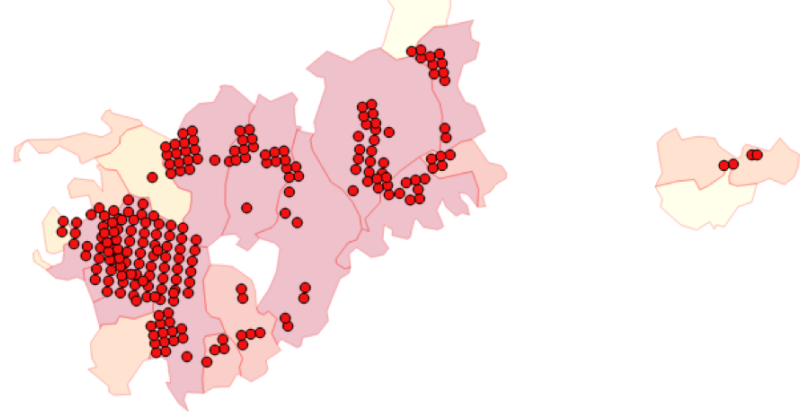

Figure 5. Identified hot spots based on VIIRS imagery. The polygons are commune boundaries and the colour code reflects the number of hot spots found within the commune.
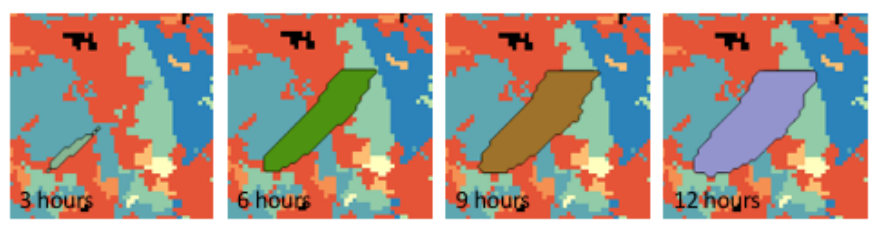

Figure 6. An example model output for the spread of wildfire based on the European Fuel Map and forecast weather conditions. The coloured polygons represent the spread of wildfire in 3 hour intervals. The background is colour coded fuel map.

on fire spread models. Figure 6, shows an example of such an output based on the forecasted weather at the time the model was run. The background shows the European fuel map that is necessary for the calculation of wildfire spread. Not all input data could be presented in the figure, e.g. DEM and fuel moisture. What is shown are outputs for the model running over 12 hours at three hour intervals. Such maps are produced automatically when a region and point of ignition is provided. This is provided when the authorities activate a disaster response. All the input data is automatically acquired and updated as new forecasts are made available.

Further help during a wildfire crisis is provided by information relating to the identification of where the most vulnerable people are located as well as important infrastructures (figure 7). This can be used for helping with evacuation plans and fire fighting. As described in the methodology, there are a number of input layers ingested including population details. With these layers in hand, authorities can tell when specific communities are in danger. In the future, natural protected habitat can also be introduced into the layers to further enhance the output wildfire risk impact maps. Note, that together with UNESCO, the system will be monitoring for potential wildfire dangers around their heritage sites.

Areas burned by wildfires can be significant and EO 


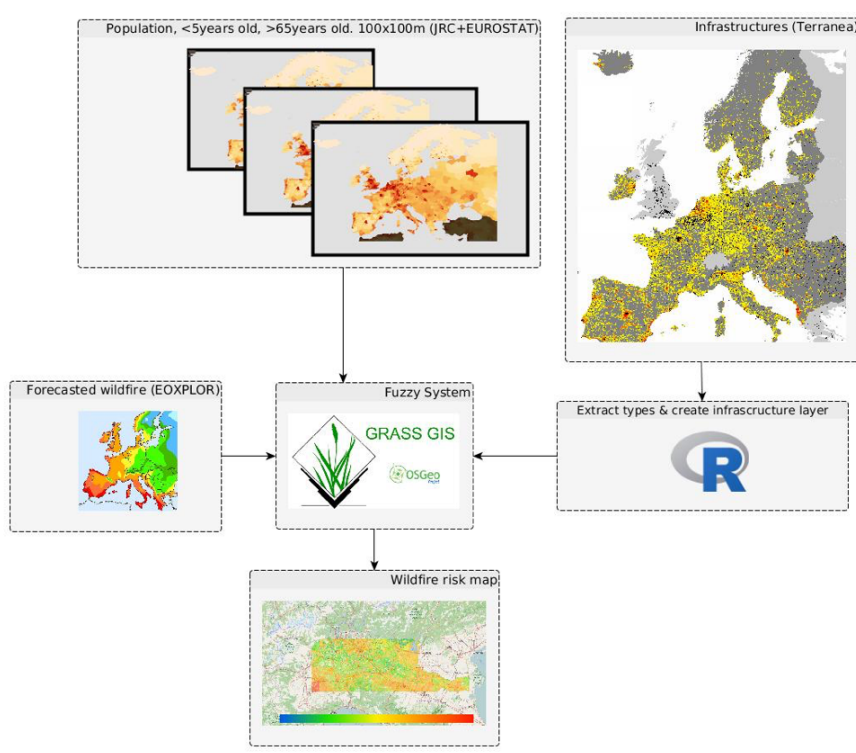

Figure 7. The risk impact raster map is computed in GRASS GIS using a fuzzy methodology pipeline, sensitivity layers: population, infrastructures and hazard forecast of wildfire. (FBK, the output and how it is generated in more detail you can find above, under process description)

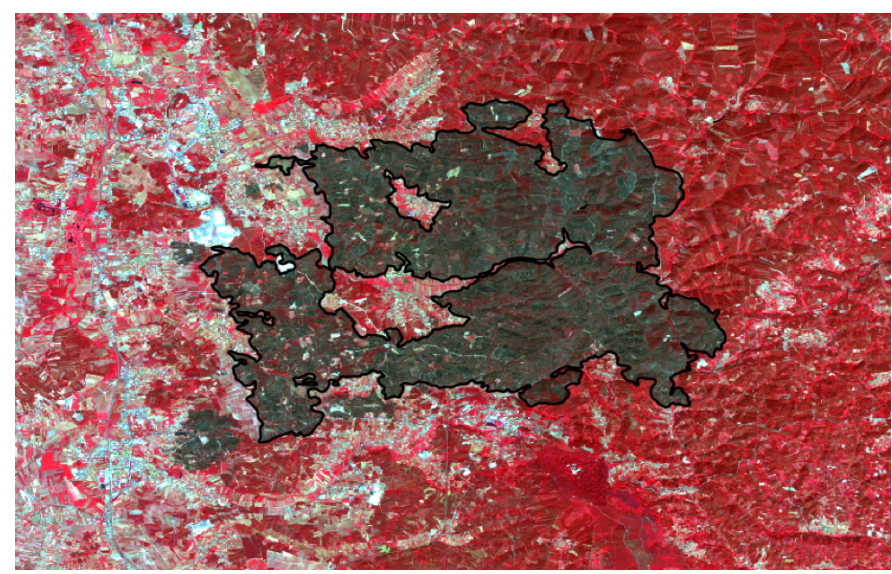

Figure 8. Burned Area Map produced using Copernicus Sentinel-2 imagery. The black outline represents the result of the automated burned area mapping. The blackened area shows charred vegetation in the post-fire false-color image $(8,4,3)$.

imagery is one of the best ways to map it (8). Such information would be useful for wildfire spread modeling if it is available during the crisis. However, as mentioned previously, mapped burned areas are necessary for the postwildfire period to understand the devastation as well as plan for other potential natural disasters such as landslides.

The wildfire monitoring and emergency management system is a complex system able to provide automatically early warnings, information acquired through crowdsourcing, sophisticated modeling results, and decision support to the authorities dealing with wildfire crises.

\section{DiscusSiOn AND FUtURE WORK}

In the context of the I-REACT project, wildfire disaster services are being developed. The presented wildfire monitoring for emergency management service shows the huge amounts of data and information that needs to be processed as well as the variety of sources, including crowdsourced and EO, in order to help the authorities reduce human as well as economic losses due to wildfire disasters.

While the system itself is still under development, the team will be presenting live demonstrations to authorities in order to test the system in the field as well as to get valuable feedback from the experts and crisis responders that have the knowledge, experience and expertise to fight wildfires. Note that the wildfire system at this time is meant to run alongside current wildfire crisis management in order provide new information and potential new insights into successfully battling such fires. Currently, the responsible authorities have wildfire emergency response plans in place that must be followed. The proposed DSS builds on those plans.

During the in-field demonstrations that we have planned for the summer of 2018, the team will be collecting valuable information from the wildfire emergency responders in order to assess the advantages and disadvantages of the system that was designed.

\section{CONClusion}

The ability today to gather data and information from a wide variety of sources, from people on the ground to Earth Observing satellites in near-Earth orbit provides a fantastic opportunity for data science in the context of emergency management. The wildfire monitoring and emergency management system presented tries to tie meaningful data and information to help in the management of wildfire crisis and reduce losses related to wildfires. From a data science perspective, the system has integrated the following relevant technologies in a modular architecture:

- Big data analytics;

- Decision support;

- Crowdsourcing for both education and information gathering;

- Social media monitoring for wildfire confirmation;

- Wildfire hazard nowcasting and forecasting;

- Wildfire risk map production.

Based on the current modular system, it is expected that as new algorithms and data sources become available, they will be easily adopted and ingested into the system to help improve wildfire crisis management.

\section{ACKNOWLEDGMENT}

This work was partially founded by the European Union through the I-REACT project (H2020-DRS-1-2015), grant agreement n.700256. The authors would also like to thank 
the other project partners who indirectly made this work possible.

\section{REFERENCES}

[1] Intergovernmental Panel on Climate Change (IPCC). Climate Change 2013: The Physical Science Basis. Contribution of Working Group I to the Fifth Assessment Report of the Intergovernmental Panel on Climate Change. http://www.climatechange2013.org/report/full-report. 2013.

[2] Harvard School of Engineering and Applied Sciences, Wildfire seasons by 2050 Wildfire study, [Online]: https://www.seas.harvard.edu/news/2016/06/fort-mcmurraywildfires-indication-of-what-s-to-come. 2016.

[3] Harvard School of Engineering and Applied Sciences, Wildfires projected to worsen with climate change, [Online]: www.seas.harvard.edu/news/2013/08/wildfires-projectedworsen-with-climate-change. 2013.

[4] Joint Research Centre (JRC) of the European Commission and European Environment Agency (EEA) Seasonal Severity Rating (SSR). [Online]: https://www.eea.europa.eu/data-andmaps/indicators/forest-fire-danger-2/assessment . 2016.

[5] Lugt D., Improving GLAMEPS wind speed forecasts by statistical postprocessing. 2013. Available: http://www.knmi.nl/bibliotheek/knmipubIR/IR2013-03.pdf

[6] Wilks, D., Extending logistic regression to provide fullprobability-distribution MOS forecasts. Meteorological Applications 16: 361-368. doi: 10.1002/met.134. 2009.

[7] Schroeder, W., Oliva, P., Giglio, L., and Csiszar, I. A.. The New VIIRS $375 \mathrm{~m}$ active fire detection data product: algorithm description and initial assessment. Remote Sensing of Environment, 143, 85-96. doi:10.1016/j.rse.2013.12.008. 2014.

[8] Justice, C. O., L. Giglio, et al. MODIS-Derived Global Fire Products. Land Remote Sensing and Global Environmental Change. B. Ramachandran, C. O. Justice and M. J. Abrams, Springer New York. 11: 661-679. doi:10.1007/978-1-44196749-7_29. 2011.

[9] Burgan, Robert E. and Rothermel, Richard C. BEHAVE: Fire Behavior Prediction and Fuel Modeling System - FUEL Subsystem. A publication of the National Wildfire Coordinating Group. PMS 439-1. NFES 0275. 1984.

[10] Xu, Jianping. Simulating the spread of wildfires using a geographic information system and remote sensing, Ph. D. Dissertation, Rutgers University, New Brunswick, New Jersey. 1994.

[11] Araya-Muoz, D., Metzger, M. J., Stuart, N., Wilson, A. M. W., Carvajal, D.. A spatial fuzzy logic approach to urban multihazard impact assessment in Concepcin, Chile. Science of the Total Environment, 576, 508?519. 2017.

[12] Keating, A., K. Venkateswaran, M. Szoenyi, K. Macclune, R. Mechler. From event analysis to global lessons: disaster forensics for building resilience. Natural Hazards and Earth System Sciences Discussions. 1-25. 10.5194/nhess-2016-52. 2016.
[13] Roy, D.P., Jin, Y., Lewis, P.E., Justice, C.O. Prototyping a global algorithm for systematic fire-affected area mapping using MODIS time series data.Remote Sensing of Environment, 97:137-162. 2005.

[14] Chen X., Vogelmann J. E., Rollins M., Ohlen D., Key C. H., Yang L., Huang C., Shi H. Detecting post-fire burn severity and vegetation recovery using multitemporal remote sensing spectral indices and field-collected composite burn index data in a ponderosa pine forest. International Journal of Remote Sensing, 32(23), 7905-7927. 2011.

[15] Veraverbeke S., Harris S., Hook S. Evaluating spectral indices for burned area discrimination using MODIS/ASTER (MASTER) airborne simulator data. Remote Sensing of Environment. 115(10), 2702-2709. 2011.

[16] C. Rossi, W. Stemberger, C. Bielski, G. Zeug, N. Costa, D. Poletto, E. Spaltro, and F. Dominici, "Coupling Crowdsourcing, Earth Observations, and E-GNSS in a Novel Flood Emergency Service in the Cloud," IEEE IGARSS. 2015. 